\title{
Structural Representation: Reducing Multi-Modal Image Registration to Mono-Modal Problem
}

Keyvan Kasiri

David A Clausi

Paul Fieguth
Vision \& Image Processing Research Group, Systems Design Engineering, University of Waterloo, ON, Canada

\section{Abstract}

Registration of multi-modal images has been a challenging task due to the complex intensity relationship between images. The standard multi-modal approach tends to use sophisticated similarity measures, such as mutual information, to assess the accuracy of the alignment. Employing such measures imply the increase in the computational time and complexity, and makes it highly difficult for the optimization process to converge. The presented registration method works based on structural representations of images captured from different modalities, in order to convert the multimodal problem into a mono-modal one. Two different representation methods are presented. One is based on a combination of phase congruency and gradient information of the input images, and the other utilizes a modified version of entropy images in a patch-based manner. Sample results are illustrated based on experiments performed on brain images from different modalities.

\section{Introduction}

Image registration is the process of aligning images by finding the correct spatial transformation between corresponding elements and structures in images. In medical imaging applications, registration of images acquired from different modalities helps clinicians in diagnosis and computer-aided surgery [1]. Because of the intensity variations originated from different imaging techniques, the registration task is becoming more difficult.

To deal with this problem, a key issue is to define an appropriate similarity measure robust to those intensity variations. Traditionally, measuring statistical dependency is measured using similarity measures, such as mutual information (MI) [2]. However, these measures would be problematic in those cases with complex and spatially dependent intensity relations [3]. Structural information has been recently utilized to transform multi-modal to mono-modal registration $[4,5]$. Reducing the multi-modal problem to a monomodal one results in using simple L1 or L2 distance metrics that are computationally less expensive than statistical or structural similarity measures. Here, two approaches are presented for extracting structural representation from images of different modalities.

\section{Methodology}

The problem of registering two images $I_{m}, I_{f}$, as the moving and fixed image, is defined by finding the space transformation , $T$, of moving image, that minimizes the dissimilarity (distance) $D$ between the two images. Here, a structural representation, $R$, of images is aimed to reduce the problem of multi-modality to a monomodal one. So, the registration problem will be formulated as

$$
\hat{T}=\underset{T}{\operatorname{argmin}} D\left(R_{f}, T\left(R_{m}\right)\right),
$$

where $R_{f}$ and $R_{m}$ are the representations for $I_{f}$ and $I_{m}$, respectively.

Two approaches are proposed to create the structural representation of images captures from different modalities.

Approach 1: In this approach, we use phase congruency (PC) which provides a simple model to imitate the human visual system for detecting and identifying features in an image [6]. The multiscale complex wavelet representation of image, $\left(I_{P C}\right)$, is computed using an over-complete Log-Gabor complex wavelet transform. As complementary information, gradient magnitude of image, $\left(I_{G M}\right)$, is extracted to encode the contrast information. The combination strategy to form the representation is propose as follows.

$$
J(\mathbf{x})=I_{G M}^{\alpha}(\mathbf{x}) \cdot I_{P C}^{\beta}(\mathbf{x}),
$$

where $\alpha$ and $\beta$ are constant parameters that are used to adjust the importance of PC and edge information.
Approach 2: In this approach, structural features are obtained from a modified version of entropy image in a patch-based paradigm. The patch entropy is modified by weighting the patch intensity histogram using a Gaussian kernel in order to spatially constrain the contribution of each pixel in the patch entropy. Also, a function $f$ is employed to increase the contribution of pixels with lower probability in the patch and weaken the pixel contribution in the smooth areas. The new formulation for the patch entropy, $\tilde{H}$, with the Gaussian mask $G$ and the patch histogram $p$ defined in the intensity mapping $\mathcal{I}$ can be expressed as

$$
\tilde{H}\left(I\left(P_{x}\right)\right)=-\sum_{i \in \mathcal{I}} G(x) p(I(x)=i) \log (f(p(I(x)=i))) .
$$

Once the structural representation is available, the registration task can be performed in an optimization procedure by using a simple distance measure such as sum of squared distance (SSD) to measure the alignment accuracy.

\section{Results}

An example of structural representation for different modes of magnetic resonance (MR) images are shown in Fig. 1. In this figure, the method to extract the structural features is the second approach based on modified entropy. As can be seen, structures extracted from different modes of MR images are independent of original intensity mappings and present similar structures in all modes.

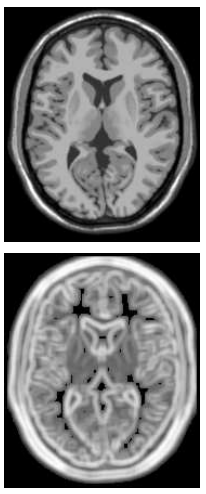

$\mathrm{T} 1$

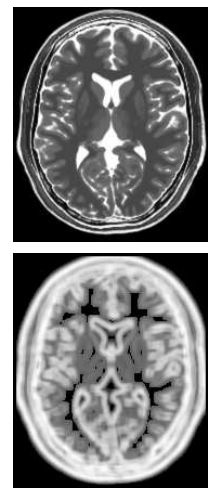

T2

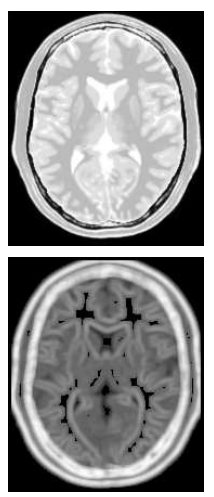

PD
Fig. 1: Structural representation for different MR modes. The first row shows a slice of brain scans in T1, T2, and PD modes. Second row shows the representation associated with the first row images.

\section{References}

[1] Crum, W. R., Hartkens, T. and Hill, D. L. G. Non-Rigid Image Registration: Theory and Practice. British Journal of Radiology (2004).

[2] Pluim, J. P. W., Maintz, J. B. A. and Viergever, M. A. MutualInformation-Based Registration of Medical Images: A Survey. IEEE Transactions on Medical Imaging (2003).

[3] Keller, Y. and Averbuch, A. Multisensor Image Registration via Implicit Similarity. IEEE Transactions on Pattern Analysis Machine Intelligence (2006).

[4] Kim, Y. S., Lee, J. H., and Ra, J. B. Multi-Sensor Image Registration Based on Intensity and Edge Orientation Information. Pattern Recognition (2008).

[5] Wachinger, C. and Navab, N. Entropy and Laplacian Images: Structural Representations for Multi-Modal Registration. Medical Image Analysis (2012)

[6] Kovesi, P. Image Features from Phase Congruency. Videre: Journal of Computer Vision Research (1999). 